\title{
IV. Die pulsatorische Erschütterung des Kopfes (das sogenannte Musset'sche Symptom).
}

\author{
Von Dr. B. Herzog in Mainz.
}

Beim Galvanisiren am Kopfe war mir schon seit Jahren eine rhythmische Erschütterung des Kopfes aufgefallen, welche sich bei näherem Zusehen als dem Pulse synchron herausstellte. Der Kopf machte um seine Frontalaxe eine kleine Zuckung nach hinten (das Gesicht wurde also ein wenig gehoben), um gleich wieder nach vörn zu sinken, hin und wieder war auch bei genauem Zusehen in der ersten Phase zuerst eine Bewegung nach links und dann eine kleine Schwankung nach rechts wahrzunehmen: die Erscheinung stand offenbar in Zusammenhang mit dem Pulse und die letzterwähnte Variante war eine Folge des direkten Ursprunges und des geradlinigen Verlaufes der Arteria carotis sin. Am besten war die Erschütterung wahrzunehmen, wenn man an dem zu ruhigem Sitzen aufgeforderten Patienten auf einen Vorhang, die Tapete vorbeifixirte, in manchen Fällen war sie aber so deutlich, dass man sie auch bei oberflächlicher Betrachtung wahrnehmen und an den Zuckungen den Puls zählen konnte. Am allerbesten sah man sie dann bei Damen, die auf ihrem Hute eine aufrecht stehende Feder hatten.

Das Rhythmische der Bewegung schloss Verwechselung mit Tremor aus.

Im Exspirium trat sie übrigens deutlicher zu Tage, als während der Inspiration.

Ich fand nun vor einiger Zeit schon, dass dieses Phänomen längst von den Franzosen als Musset'sches Symptom bei Aortenaneurysma und Aorteninsuffizienz beschrieben wurde. Zuletzt stellte meines Wissens Charles Valentino ${ }^{1}$ ) 14 Fälle und einen von Frenkel ${ }^{2}$ ) bei Pleuritis beobachteten Fall zusammen und erklärte es durch Blutstauung, als Zeichen des mit der Diastole zusammenhängenden Blutrefluxes. Der klinische Werth liegt nach dem Autor darin, dass es "bei Ausschluss jeder nervösen Störung (Tic douloureux etc. [?]) ... unmittelbar die Aufmerksamkeit des Arztes auf die Herzgegend lenken muss und für ihn das erste Zeichen einer bis dahin latenten oder nicht erkannten Herzaffektion bilden kann" (Münchener medizinische Wochenschrift). Ich fand es nun bei einem Falle von Aortenaneurysma (R.) sehr deut-

*) Anmerkung bei der Correktur: Patient konnte in der Zwischenzeit geheilt entlassen werden. Wie wir angenommen haben, nahm die Urinentleerung aus der ursprünglichen Fistel immer mehr ab; die Fistel schloss sich darauf ohne Anfrischung. Nachdem auch der Blasenkatarrh ganz wesentlich gebessert war, sodass die Urinentleerung wieder per vias naturales ohne Beschwerden erfolgen konnte, wurde auch der Schlauch entfernt; wenige Tage darauf war die Schrägfistel geschlossen.

1) Revue de méd., Mai 1902.

2) Presse méd. VIII 94, 14. Nov. 1900. 
lich, bei einem zweiten (A.) angedeutet; bei drei Fällen von Aorteninsuffizienz (W. B. und Gr.) sehr deutlich, bei einem vierten (Gr. . .) nur angedeutet.

Das Vorkommen bei Aorteninsuffizienz hatte eigentlich nichts Auffallendes. Es ist uns bekannt, dass bei dieser Herzaffektion die peripheren Arterien erweitert und verlängert sind, sodass kleine sonst unfühlbare (Fingerarterien u. a.) fühlbar pulsiren. Dazu kommt, dass, während also das Blut in der Systole in grösserer Menge und unter hohem Drucke in die Arterien strömt, der Druck während der Diastole rasch absinkt. Dass durch Uebertragung dieser starken Blutdruckschwankung pulsatorische Bewegungen des Gehirns und damit Erschütterungen des Kopfes entstehen können, ist nicht wunderbar. Neu dürfte nur sein, dass der Schädel keine diffuse Erschütterung erfährt, sondern eine Art Pulskurve beschreibt, wie etwa der Unterschenkel, wenn im Sitzen die Kniekehle des einen Beines auf der Kniescheibe des anderen ruht [Placzek ${ }^{1}$, Crispino ${ }^{2}$ )].

Durchaus nicht die gleichen Verhältnisse gelten für das Aneurysma; denn hier erreicht die zur Aufrechterhaltung des Kreislaufes entstehende Hypertrophie des linken Ventrikels durchaus nicht immer den Grad, dass sie einen gesteigerten Blutdruck im Arteriensystem bewirkt.

Nun fand ich das Phänomen aber auch recht ausgesprochen in einer Reihe anderer Fälle: bei einigen Unfallpatienten mit Zeichen von Atherom, bei. Alkoholisten, hier und da bei interstitieller Nephritis, endlich bei Hypertrophia ventriculi sin. bei Atherom.

Die meisten dieser Patienten zeigten Drucksteigerungen im Arteriensystem von $130 \mathrm{~mm}$ bis $\mathrm{zu} 220 \mathrm{~mm} \mathrm{Hg}^{3}$ ). Aber während einerseits manche Patienten dieser Art bei gesteigertem Drucke das Symptom vermissen liessen oder nur angedeutet zeigten (auch der oben erwähnte Fall G. zeigte es nur "angedeutet" bei $150 \mathrm{~mm} \mathrm{Hg}$ ), boten andere mit normalem oder unternormalem Drucke es recht deutlich dar.

Also musste man zur Erklärung des Phänomens eine lokale Drucksteigerung entweder auf dem Boden des Atheroms oder durch Stauung, heranziehen. Dafür sprechen auch die beiden Fälle von Aneurysma, welche sehr geringen Blutdruck haben (R. $110 \mathrm{~mm}$, A. nur digital gemessen). Auf die begünstigende Rolle der „Stauung“ wies endlich die oben erwähnte Thatsache des Undeutlicherwerdens während der Inspiration mit ihrer das Blut ansaugenden Wirkung und das Deutlicherwerden während der exspiratorischen Phase hin.

Sehr instruktiv waren in dieser Richtung zwei Fälle von Lues cerebri ohne Blutdrucksteigerung an der Radialis, deren einer einen auswärtigen Collegen betraf. Dieser Herr, bisher gesund, consultirte mich vor einigen Jahren wegen einiger Schwindelanfälle. Die Untersuchung fiel zunächst negativ aus, erst das Musset'sche Phänomen liess mich nochmals das Herz untersuchen, welches eine deutliche Accentuation des zweiten Aortentones zeigte. Diese beiden Erscheinungen führten dazu, dass ich bei dem im mittleren Lebensalter stehenden Herrn die Anamnese vervollständigte, wobei sich herausstellte, erstens, dass die Schwindelanfälle sehr schwer gewesen waren und zweitens, dass Lues vorangegangen war. Die Bestätigung meines Verdachtes und die völlige Heilung unter entsprechender Medikation ergaben das Weitere.

Die Stauung kann aber nicht in den Lymphbahnen ihren Sitz haben, denn ich vermisste das Phänomen bei Tumor cerebri in einem Falle und Prof. Sommer ${ }^{4}$ ) in zwei Fällen.

Bei erregten Neurasthenikern sah ich es bei gesteigertem, normalem und niedrigem Blutdrucke (bei $70-100 \mathrm{~mm} \mathrm{Hg}$ ) sehr oft - wenn auch nie sehr ausgesprochen - und deshalb glaube ich, als dritten und wichtigsten Faktor für seine Entstehung mechanische Momente verantwortlich machen zu sollen, indem (wie bei dem Kniekehlenphänomen) der an der Arterienkrümmung eintretende Verlust der dahinstürzenden Blutsäule an Bewegungsenergie eine Rolle spielt. Vielleicht wirkt übrigens bei den zuletzt erwähnten Fällen auch der geringere Tonus der Hals- und Nackenmuskeln begünstigend mit.

1) Berliner klinische Wochenschrift 1899, No. 31

2) Centralblatt für innere Medizin 1901, No. 13.

3) Ich arbeitete mit dem Sphygmomanometer von v. Basch.

4) Dem ich für seine freundliche Mittheilung an dieser Stelle herzlichen Dank sage.
Alles in allem scheint das Phänomen bei Aortenaneurysmen und -Insuffizienz, sowie bei erheblicher Drucksteigerung aus anderen Gründen fast constant, sodann aber auch in Fällen von nur örtlicher Steigerung des Druckes hier und da vorzukommen. Für alle diese Fälle (Lues, Atherom der Hirnarterien, Unfallpatienten) wird es zuweilen semiotisch von einem gewissen Werthe sein können, indem es zu sorgfältigster Untersuchung des Cirkulationsapparates mahnt. 